Med Klin Intensivmed Notfmed $2021 \cdot 116$ : 281-294

https://doi.org/10.1007/s00063-021-00794-4

Angenommen: 1. Februar 2021

Online publiziert: 1. März 2021

(c) Der/die Autor(en) 2021

Andrej Michalsen ${ }^{1} \cdot$ Gerald Neitzke $^{2} \cdot$ Jochen Dutzmann ${ }^{3} \cdot$ Annette Rogge $^{4} \cdot$ Anna- $^{2}$ Henrikje Seidlein ${ }^{5} \cdot$ Susanne Jöbges ${ }^{6} \cdot{\text { Hilmar Burchardi }{ }^{7} \text {. Christiane Hartog }}^{8,9}$. Friedemann Nauck ${ }^{10}$. Fred Salomon ${ }^{11}$. Gunnar Duttge ${ }^{12}$. Guido Michels ${ }^{13}$. Kathrin Knochel ${ }^{14,15}$. Stefan Meier ${ }^{16} \cdot$ Peter Gretenkort $^{17} \cdot$ Uwe Janssens ${ }^{18}$

' Klinik für Anästhesiologie, Intensivmedizin, Notfallmedizin und Schmerztherapie, Klinikum Konstanz, Konstanz, Deutschland; ${ }^{2}$ Institut für Geschichte, Ethik und Philosophie der Medizin, Medizinische Hochschule Hannover, Hannover, Deutschland; ${ }^{3}$ Universitätsklinik und Poliklinik für Innere Medizin III, Universitätsklinikum Halle (Saale), Halle (Saale), Deutschland; ${ }^{4}$ Geschäftsbereichs der Medizinethik, Christian-Albrechts-Universität zu Kiel, Kiel, Deutschland; ${ }^{5}$ Institut für Ethik und Geschichte der Medizin, Universitätsmedizin Greifswald, Greifswald, Deutschland; ${ }^{6}$ Institut für Biomedizinische Ethik und Geschichte der Medizin, Universität Zürich, Zürich, Schweiz; ${ }^{7}$ Bovenden, Deutschland; ${ }^{8} \mathrm{Klinik}$ für Anästhesiologie und Intensivmedizin, Charité Universitätsmedizin Berlin, Berlin, Deutschland; ${ }^{9}$ Patienten- und Angehörigenzentrierte Versorgung (PAV), Klinik Bavaria, Kreischa, Deutschland; ${ }^{10} \mathrm{Klinik}$ für Palliativmedizin, Georg-August-Universität Göttingen, Göttingen, Deutschland; "Lemgo, Deutschland; ${ }^{12}$ Abteilung für strafrechtliches Medizin- und Biorecht, Georg-August-Universität Göttingen, Göttingen, Deutschland; ${ }^{13}$ Klinik für Akut- und Notfallmedizin, St.-Antonius-Hospital Eschweiler, Eschweiler, Deutschland; ${ }^{14}$ Kinderklinik und Kinderpoliklinik im Dr. von Haunerschen Kinderspital Kinderpalliativzentrum, Klinikum der Universität München, München, Deutschland; ${ }^{15}$ Ethik der Medizin und Gesundheitstechnologie, Technische Universität München, München, Deutschland; ${ }^{16} \mathrm{Klinik}$ für Anästhesiologie, Universitätsklinikum Düsseldorf, Düsseldorf, Deutschland; ${ }^{17}$ Simulations- und Notfallakademie, Helios Klinikum Krefeld, Krefeld, Deutschland; ${ }^{18} \mathrm{Klinik}$ für Innere Medizin und Internistische Intensivmedizin, St.-Antonius-Hospital Eschweiler, Eschweiler, Deutschland

\title{
Überversorgung in der Intensivmedizin: erkennen, benennen, vermeiden
}

\section{Positionspapier der Sektion Ethik der DIVI und der Sektion Ethik der DGIIN}

[SGB] V) existieren im deutschen Gesundheitssystem in der Patientenversorgung nebeneinander Unter-, Fehl- und Überversorgung [20, 33, 65].

Das vorliegende Positionspapier richtet den Blick auf die Überversorgung in der Intensivmedizin [14, 31, 62]. Ziel der Intensivmedizin ist es, lebensbedrohliche medizinische Krisen zu überbrücken, damit die Patientinnen nach der intensivmedizinischen Behandlung weitgehend selbstbestimmt und mit einer für sie akzeptablen Lebensqualität außerhalb der Intensivstation weiterleben können. Grundsätzlich müssen für jede (intensiv)medizinische Behandlung erstens eine Indikation und zweitens eine rechtswirksame Einwilligung durch die Patientin oder ihren Stellvertreter vorliegen (sog. Zwei-Säulen-Modell; [17]). Die Behandlungsteams müssen also - auch wiederholt - die jeweilige Prognose bewerten und abwägen, welche der häufig komplexen Behandlungsmaßnahmen medizinisch sinnvoll für die individuelle Patientin unter Berücksichtigung ihres Willens und ihrer Wertvorstellungen angewandt werden können und dürfen. Es stellt somit eine große Herausforderung und Verantwortung dar, den jeweils angemessenen Behandlungsumfang $\mathrm{zu}$ bestimmen.

Überversorgung bezeichnet Behandlungsmaßnahmen, die nicht angemessen sind, weil sie $\mathrm{zu}$ keiner für die $\mathrm{Pa}$ tientin bedeutsamen Verbesserung der (Über-)Lebensdauer oder Lebensqualität führen, mehr Schaden als Nutzen verursachen und/oder von Patientinnen nicht gewollt werden $[51,56]$. Überversorgung ist also nicht von Bedarf und Bedürfnis der Patientin in ihrer individuellen 


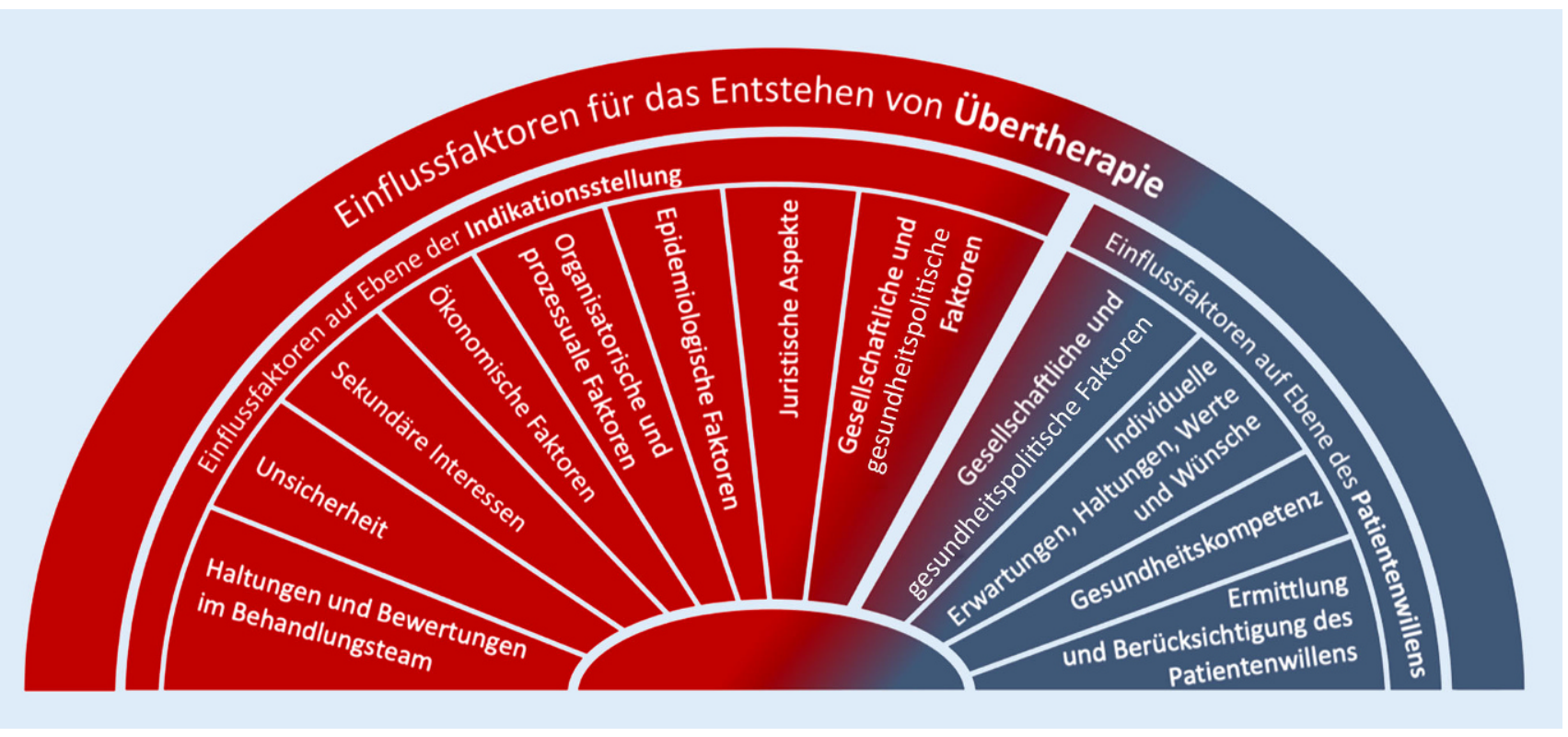

Abb. 1 ॥ Einflussfaktoren für das Entstehen von Übertherapie sind vielfältig und finden sich aufEbene der Indikationsstellung und auf der Ebene des Patientenwillens

Krankheitssituation, sondern durch andere Motive veranlasst. Überversorgung kann mit hohen Belastungen und Risiken für die betroffenen Patientinnen, ihre Familien und die Behandlungsteams verbunden sein; sie kann Leiden und Trauer verursachen oder verlängern sowie $\mathrm{zu}$ Gewissensnot, „moral distress“, Burnout und Personalabwanderung beitragen [40, 44]. Überversorgung schadet der Allgemeinheit, weil die dadurch gebundenen Ressourcen für andere Zwecke nicht mehr zur Verfügung stehen (Opportunitätskosten). Außerdem kann eine Überversorgung in der Intensivmedizin in allen nachfolgenden Bereichen des Gesundheitssystems eine ungerechtfertigte Versorgungskaskade verursachen. Insofern ist es dringlich geboten, Überversorgung auch in der Intensivmedizin zu erkennen und zu vermeiden. Darüber hinaus ist es wichtig $\mathrm{zu}$ verdeutlichen, dass Therapielimitierungen nicht das Ende aller medizinischen und pflegerischen Behandlung bedeuten. Angebote der palliativmedizinischen Behandlung und Begleitung sind darauf ausgerichtet, körperliches und psychosoziales Leid zu lindern und Patientinnen und ihre An- und Zugehörigen in der letzten Lebensphase nicht allein zu lassen.

\section{Ursachen für Überversorgung}

Gesellschaftliche Wertvorstellungen und Übereinkünfte, Organisations- und Versorgungsstrukturen des Gesundheitswesens, demographische und medizintechnische Entwicklungen, rechtliche Regelungen, kommerzielle Interessen und insbesondere die individuelle ArztPatienten-Beziehung beeinflussen mit unterschiedlicher Wirkkraft den Behandlungsumfang. Diese unterschiedlichen Faktoren können damit auch Überversorgung begünstigen oder vermeiden. Während die beiden Säulen „Indikation“ und „Patientenwille“ für die Behandlungsentscheidung gleichermaßen relevant sind, beeinflussen sie Überversorgung in unterschiedlicher Weise (• Abb. 1).

\section{Einflussfaktoren bei der Indikationsstellung}

\section{Haltungen und Bewertungen innerhalb des Behandlungsteams} Indikationsstellungen werden im Alltag nicht nur von objektiven Fakten, sondern auch von persönlichen, intuitiven und emotionalen Bewertungen zur Sinnhaftigkeit der jeweiligen medizinischen Maßnahmen bestimmt [48]. Dazu gehören auch die etabliert vermittelten und erlernten Fähigkeiten zur kritischen Reflexion, die Prägung durch die Fachzugehörigkeit und das berufliche Umfeld, das Menschenbild, die Religiosität und andere soziokulturelle Faktoren [17, 74].

Vielfach stellen persönliche Faktoren der Behandelnden substanzielle Ursachen für den Versorgungsumfang dar [34, 65]. Beispielsweise wird die grundsätzlich positive Einstellung, Leben retten und erhalten zu wollen, dann zu einer negativen und gefährlichen Haltung, wenn die Rettung auch in erkennbar aussichtslosen Fällen versucht wird. Selbstüberschätzung und unbewusste Allmachtsphantasien können dabei eine Rolle spielen. Es fällt oft leichter, alle zur Verfügung stehenden Mittel unreflektiert anzuwenden, als differenziert und individuell nach Therapiezielen und Erfolgsaussicht zu fragen („Retten um jeden Preis“, „rule of rescue“ [29]). Die Tabuisierung von Sterben und Tod auch innerhalb der Behandlungsteams und das Erleben von Tod als persönliches Versagen können diesen Effekt verstärken. Eine „schöngeredete“ Einschätzung der Prognose führt in diesen Fällen zu letztlich sinnlosen Therapiebemühungen.

Forschungsergebnisse aus der Psychologie der Entscheidungsfindung benennen unwillkürliche Entscheidungsfehler, 
Med Klin Intensivmed Notfmed 2021 • 116:281-294 https://doi.org/10.1007/s00063-021-00794-4

(c) Der/die Autor(en) 2021

\section{A. Michalsen · G. Neitzke · J. Dutzmann · A. Rogge · A.-H. Seidlein · S. Jöbges · H. Burchardi · C. Hartog · F. Nauck · F. Salomon · G. Duttge · G. Michels $\cdot$ K. Knochel $\cdot$ S. Meier $\cdot$ P. Gretenkort $\cdot$ U. Janssens \\ Überversorgung in der Intensivmedizin: erkennen, benennen, vermeiden. Positionspapier der Sektion Ethik der DIVI und der Sektion Ethik der DGIIN}

\section{Zusammenfassung}

Ungeachtet der sozialgesetzlichen Vorgaben existieren im deutschen Gesundheitssystem in der Patientenversorgung nebeneinander Unter-, Fehl- und Überversorgung. Überversorgung bezeichnet diagnostische und therapeutische Maßnahmen, die nicht angemessen sind, da sie die Lebensdauer oder Lebensqualität der Patienten nicht verbessern, mehr Schaden als Nutzen verursachen und/oder von den Patienten nicht gewollt werden. Daraus können hohe Belastungen für die Patienten, deren Familien, die Behandlungsteams und die Gesellschaft resultieren. Dieses Positionspapier erläutert
Ursachen von Überversorgung in der Intensivmedizin und gibt differenzierte Empfehlungen zu ihrer Erkennung und Vermeidung. Zur Erkennung und Vermeidung von Überversorgung in der Intensivmedizin erfordert es Maßnahmen auf der Mikro-, Meso- und Makroebene, insbesondere die folgenden: 1) regelmäßige Evaluierung des Therapieziels im Behandlungsteam unter Berücksichtigung des Patientenwillens und unter Begleitung von Patienten und Angehörigen; 2) Förderung einer patientenzentrierten Unternehmenskultur im Krankenhaus mit Vorrang einer qualitativ hochwertigen
Patientenversorgung; 3) Minimierung von Fehlanreizen im Krankenhausfinanzierungssystem gestützt auf die notwendige Reformierung des fallpauschalbasierten Vergütungssystems; 4) Stärkung der interdisziplinären/interprofessionellen Zusammenarbeit in Aus-, Fort- und Weiterbildung; 5) Initiierung und Begleitung eines gesellschaftlichen Diskurses zur Überversorgung.

\section{Schlüsselwörter}

Therapie am Lebensende - Behandlungsausmaß. Patientenversorgung - Therapieziel . Ärztliche Indikation · Patientenwille

\section{Overtreatment in intensive care medicine-recognition, designation, and avoidance. Position paper of the Ethics Section of the DIVI and the Ethics section of the DGIIN}

\begin{abstract}
Despite social laws, overtreatment, undertreatment, and incorrect treatment are all present in the German health care system. Overtreatment denotes diagnostic and therapeutic measures that are not appropriate because they do not improve the patients' length or quality of life, cause more harm than benefit, and/or are not consented to by the patient. Overtreatment can result in considerable burden for patients, their families, the treating teams, and society. This position paper describes causes of overtreatment in intensive care medicine and
\end{abstract}

makes specific recommendations to identify and prevent it. Recognition and avoidance of overtreatment in intensive care medicine requires measures on the micro-, mesoand macrolevels, especially the following: (1) frequent (re-)evaluation of the therapeutic goal within the treating team while taking the patient's will into consideration, while simultaneously attending to the patients and their families; (2) fostering a patientcentered corporate culture in the hospital, giving priority to high-quality patient care; (3) minimizing improper incentives in health care financing, supported by reform of the reimbursement system that is still based on diagnose-related groups; (4) strengthening of interprofessional co-operation via education and training; and (5) initiating and advancing a societal discourse on overtreatment.

Keywords

End of life care - Patient care - Extent of treatment · Therapeutic goal · Patient preference die zur Übertherapie führen können [58], beispielsweise:

"Sunk cost effect": Behandler halten an einer einmal getroffenen Entscheidung fest, weil sie bereits viel investiert haben und diese Investition nicht "umsonst" gewesen sein soll. Dieser Effekt kann sich so weit verstärken, dass immer mehr und immer sinnlosere Aktionen unternommen werden.

"Omission bias" („Unterlassungseffekt"): Stirbt eine Patientin, nachdem das Behandlungsteam entschieden hat, den Behandlungsumfang zu limitieren, erscheint dies subjektiv schlimmer, als dass die Pa- tientin unter einer fortlaufenden Therapie verstirbt.

\section{Unsicherheit}

Übertherapie kann auch durch Unsicherheit verursacht sein, einerseits durch mangelnde Kenntnis des aktuellen medizinischen Wissensstands sowie der ethischen und juristischen Rahmenbedingungen, insbesondere hinsichtlich der „end-of-life care“ $[17,65]$. Andererseits ist Medizin bei der Einschätzung der Therapiewirksamkeit oder hinsichtlich prognostischer Aussagen für den individuellen Einzelfall immer von Unsicher- heiten geprägt [16]. Diese Unsicherheit muss vom Behandlungsteam für den Einzelfall wahrgenommen, eingeordnet, bewertet und in Entscheidungsprozesse einbezogen werden, um Fehlversorgung generell $\mathrm{zu}$ reduzieren [81]. Unsicherheiten müssen insbesondere im ArztPatienten-Gespräch ehrlich und empathisch angesprochen werden.

\section{Konflikte bei sekundären Interessen}

Das primäre ärztliche Interesse muss grundsätzlich die bestmögliche Behandlung jeder Patientin sein [28]. Auf der individuellen Ebene können Konflik- 


\section{Leitfragen}

Entscheidungsgrundlage

1. Wie lautet das übergeordnete Therapieziel?

2. Haben sich seit der letzten Reevaluation Befunde oder Änderungen/Erkenntnisse hinsichtlich des Patientenwillens ergeben, vor deren Hintergrund das Therapieziel kritisch überdacht und ggf. neu definiert werden muss?

Identifikation von Überversorgung auf der Indikationsebene

3. Hat jede unserer geplanten diagnostischen Maßnahmen eine Konsequenz, mit der wir dem Erreichen des Therapieziels näherkommen oder aus der wir ein neues Therapieziel ableiten würden?

4. Ist jede einzelne unserer geplanten oder laufenden therapeutischen Maßnahmen geeignet, notwendig und angemessen, um das Therapieziel zu erreichen?

Identifikation von Überversorgung auf Ebene des Patientenwillens

5. Entsprechen unsere geplanten und laufenden diagnostischen und therapeutischen Maßnahmen (weiterhin) dem Patientenwillen?

te bei sekundären Interessen, wie die Gewährung oder Annahme finanzieller Vorteile (z. B. Bonus- oder Beraterverträge) oder die Verbesserung des sozialen Status (z. B. Wunsch nach Anerkennung oder Karrierestreben), das Risiko einer Überversorgung erhöhen [5, 38].

\section{Überbetonung ökonomischer Faktoren}

Die Einführung des deutschen Fallpauschalensystems im Jahr 2002 (basiert auf „diagnosis-related groups“, DRG) hat den ökonomischen Druck auf die Krankenhäuser politisch intendiert verschärft [12]. Dabei wurde versäumt, die Einführung von Fallpauschalen im Krankenhauswesen mit Strukturveränderungen zu verbinden [61, 72]. Die Ökonomisierung deutscher Krankenhäuser hat dazu geführt, dass Erlösoptimierung $\mathrm{zu}$ einem wesentlichen Unternehmensziel geworden ist. Dieser ökonomische Druck beeinflusst die Intensität der Leistungen und damit implizit auch die Behandlungsentscheidungen. Bei privaten Krankenhausträgern ist besonders problematisch, dass dort Gewinne erwirtschaftet werden sollen, die zumindest

\section{Leitfragen}

1. Verstehen wir die aktuellen Erkrankungsbilder und Verdachtsdiagnosen?

2. Falls nein, haben wir rechtzeitig weitergehende Expertise eingeholt?

potenziell dem Gesundheitssystem entzogen und zweckentfremdet Aktionären zugeführt oder in gesundheitsfernen Bereichen investiert werden können [75]. Die Bundesländer hingegen kommen ihrer Verpflichtung zur Übernahme der Investitionsmittel für die Krankenhäuser nur unzureichend nach. Dies schafft Einfallstore für Überversorgung durch Erzwingen eines betriebswirtschaftlich begründeten Verhaltens, führt in der Fläche $\mathrm{zu}$ ineffizienten Versorgungsstrukturen und provoziert eine unzulässige Quersubventionierung aus Beitragsmitteln der gesetzlichen Krankenversicherungen in Milliardenhöhe.

Das System der DRG gibt den Kliniken Anreize zu Mengensteigerungen, insbesondere bei Maßnahmen, bei deren Vergütung rechnerisch ein hoher Anteil auf Fixkosten entfällt. Operative und interventionelle Leistungen werden dabei häufig besser vergütet als konservative Behandlungsmaßnahmen oder ein ausführliches Arzt-Patienten-/-Angehörigen-Gespräch. Dies erhöht das Risiko einer Überversorgung. In den letzten Jahren stieg beispielsweise die Inanspruchnahme von Intensivtherapie jährlich mit $3 \%$ deutlich stärker als die Inanspruchnahme von Krankenhausbehandlung insgesamt $(0,8 \%)$, vor allem am Lebensende. Inzwischen erhält jede/r vierte aller Patienten, die in einem Krankenhaus versterben, eine Intensivtherapie [18].

Die per se kostenintensiven Intensivstationen sind inzwischen zentral in das System der wirtschaftlich orientierten Krankenhausunternehmen eingebettet. Insbesondere die Kodierungsmöglichkeit „intensivmedizinische Komplexbehandlung“ (OPS 8.98f.) verleitet zur exzessiven Nutzung, also Gewinnabschöpfung, ohne dass der entsprechende Behandlungsumfang tatsächlich immer erforderlich ist. Hingegen spielen Maßnahmen zur Verbesserung einer patientenorientierten Indikations- und Ergebnisqualität im aktuellen Finanzie- rungssystem keine regulierende Rolle [61].

\section{Organisatorische und prozessuale Faktoren}

Die Gewinnoptimierung wird wesentlich durch Einsparungen im Personalbereich bei gleichzeitiger Ausweitung von Leistungen erzielt. Eine Mehrbelastung des Behandlungsteams durch eine (geplante) personelle ärztliche und/oder pflegerische Unter- oder Fehlbesetzung hat erhebliche negative Auswirkungen auf die Gesundheit des Personals und die Gesundung der Patientinnen [40, 44, 52, 76]. Personalunterdeckung erfordert ein (subjektiv) selektives Auswählen von Teilaufgaben - also ggf. auch eine implizite Rationierung - und schränkt die Möglichkeit ein, das Behandlungsziel interprofessionell und interdisziplinär zu reflektieren. Dadurch wird ein unkritisches Einleiten oder Fortsetzen von Therapiemaßnahmen befördert, was einer Überversorgung Vorschub leistet $[80,82]$.

An den Schnittstellen Übergabe und Visite besteht durch mangelhaft strukturierte Durchführung und Dokumentation das Risiko von Informationsverlusten $[3,37]$. Nachfolgend kann es dann beispielsweise $\mathrm{zu}$ erneuten, nicht notwendigen oder auch patientenseits nicht gebilligten diagnostischen oder therapeutischen Maßnahmen kommen. Dies führt nicht selten einerseits zu Rückschritten im Genesungsprozess und andererseits zu längeren Krankenhausverweildauern [3] und steigert die Opportunitätskosten. Insbesondere wenn Entscheidungen zur Therapielimitierung nicht rechtzeitig getroffen und kommuniziert werden, erfolgt häufig eine sinnlose Therapieeskalation.

Das „ethische Klima“ einer Intensivstation fasst die gemeinsame Denkund Handlungsweise des Behandlungsteams zusammen. Damit beeinflusst das ethische Klima auf einer Intensivstation wesentlich und wirksam, ob

- Therapieziele frühzeitig angespro-

chen und deren Erreichbarkeit regelmäßig überprüft werden,

- die (Tages-)Therapieziele patientenrelevant sind oder lediglich der 
Hier steht eine Anzeige.

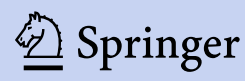


Korrektur (patho)physiologischer Parameter dienen,

- Entscheidungen zu Therapiekonzept und -umfang im Team getroffen werden,

- Therapiebegrenzungen zugelassen oder vermieden werden und

- eine patienten- und angehörigenzentrierte Versorgung stattfindet.

Das ethische Klima wird entscheidend durch Haltung und Handeln der ärztlichen und pflegerischen Leitungspersonen sowie das Miteinander der verschiedenen beteiligten Fachdisziplinen bestimmt. Ein mangelhaftes ethisches Klima begünstigt Überversorgung, ein gutes ethisches Klima hingegen begünstigt einen berufsgruppen- und fachübergreifenden Diskurs und einen individuell angemessenen Behandlungsumfang [4, $41,76]$.

\section{Juristische Faktoren}

Die Sorge vor juristischen, d. h. haftungsoder gar strafrechtlichen Konsequenzen ist seit langem als bedeutsamer Faktor für Überdiagnostik und Übertherapie bekannt. Um nicht wegen unterlassener Hilfeleistung oder eines Behandlungsfehlers belangt zu werden, scheuen nicht nur weniger erfahrene Behandelnde vor einer Verantwortungsübernahme für therapiebegrenzende Entscheidungen zurück (,juristische Indikation“; [78]) und warten zögerlich lieber auf einen „Wink des Schicksals“. Dabei birgt die Hintanstellung des Patientenwohls selbst rechtliche Risiken, von den professionsethischen Bedenken gegenüber einer solchen „Defensivmedizin“ ganz abgesehen.

Eine wesentliche Ursache hierfür ist der Umstand, dass einschlägige gesetzliche Vorschriften, juristische Begriffe und Gerichtsentscheidungen dem Behandlungsteam unzureichend bekannt sind oder nicht zutreffend interpretiert werden. Auf die Einordnung juristischer Aspekte sind (angehende) Ärztinnen bislang jedenfalls nicht ausreichend vorbereitet. Auch das Rechtssystem achtet zu wenig auf das Ziel der Orientierungssicherheit. Widerstreitende Rechtsmeinungen, mitunter gegenläufige Urteile sowie ein Hang zur Überdifferenzie- rung erzeugen elementare Unsicherheiten der Behandelnden bei Therapieentscheidungen. Das wiegt umso schwerer, als die gesellschaftliche Akzeptanz der Rechtsprechung rechtssoziologisch nicht ernsthaft evaluiert oder hinterfragt wird. Erforderliche Entscheidungen zur Therapiebegrenzung werden vor diesem Hintergrund nicht getroffen oder hinausgezögert [15].

\section{Gesellschaftliche und politische Faktoren}

In Deutschland muss bislang niemand auf die zügige und gründliche Behandlung einer schweren Erkrankung verzichten. Die ständigen technologischen und pharmakologischen Weiterentwicklungen in der Medizin, das Interesse verschiedener Institutionen, an einem wirtschaftlich attraktiven Wachstumsmarkt teilzuhaben, und die demographische Entwicklung leisten aber einer künftigen Mittelknappheit in der medizinischen Versorgung Vorschub. Des ungeachtet findet in der Öffentlichkeit bisher eine offene und vor allem transparente Diskussion über die Grenzen des medizinischen Versorgungsystems in diesem Spannungsfeld und über die Überversorgung nicht statt. Vielmehr wurde die Erwartungshaltung der Bevölkerung an eine "grenzenlose Medizin“ durch ein zunehmend gewinnorientiertes und von privaten Trägern gesteuertes Gesundheitssystem weiter befeuert.

Die Maßlosigkeit sowohl vieler Nutzer des medizinischen Versorgungssystems als auch derjenigen, die bei ihnen ungerechtfertigte Begehrlichkeiten wecken, verstärken und befriedigen, lässt allzu oft vergessen, dass Gesundheit prinzipiell ein öffentliches Gut darstellt. Die Bewahrung von Gesundheit muss solidarisch getragen, finanziert und durch ein grundsätzliches ärztliches Versprechen zur Hilfeleistung aufrechterhalten werden können $[36,45]$. Überversorgung trägt maßgeblich dazu bei, die Aufrechterhaltung einer solidarischen Gesundheitsversorgung, an der alle Patienten teilhaben können, langfristig zu gefährden.

\section{Epidemiologische Faktoren}

Gesellschaftliche Vorstellungen von Gesundheit und Krankheit, Erwartungen an die Leistungsfähigkeit der Medizin und bestehende Krankheitsdefinitionen beeinflussen sich wechselseitig. Beispielsweise fehlt gerade auch der Intensivmedizin bis heute ein altersadaptiertes Gesundheitskonzept. Jedenfalls müssen Krankheitsdefinitionen, therapierelevante Grenzwerte und diagnostische oder therapeutische Vorgehensweisen regelmäßig auf ihre Evidenz überprüft und ggf. angepasst werden. Änderungen können allerdings auch ohne Vorliegen einer ausreichenden Evidenz oder aufgrund illegitimer Interessen herbeigeführt werden. Das kann zu einer scheinbaren Zunahme von Inzidenz und Prävalenz sowie einer Überschätzung der Effektivität und Unterschätzung von Nebenwirkungen führen. Daraus lässt sich eine vermeintliche Behandlungsnotwendigkeit konstruieren. Eine dann resultierende Übertherapie nutzt ökonomischen und/oder sekundären Interessen und bewirkt jedenfalls einen ungerechtfertigten Ressourcenverbrauch und unter Umständen auch Schädigungen der Patientinnen [6, 7, 25, 26, 59].

\section{Einflussfaktoren auf den Patientenwillen}

\section{Formung des Patientenwillens}

Die Bedeutung von Gesundheit und Krankheit und die damit verbundenen Forderungen nach Behandlungsmaßnahmen sind tief verwurzelt in den Werten von Familie, von soziokultureller Prägung und Einbindung sowie von Bildungsgrad, Religion und Religiosität [27, 39, 73, 74]. Denkmuster, Verhaltensweisen und Erwartungshaltungen der Patientinnen an die moderne Medizin sowie das ständig wachsende Angebot des Gesundheitssystems beeinflussen - wissentlich oder auch unbewusst - eine Überdiagnostik und -therapie. Dazu gehören z. B. das fehlende Verständnis für eine (Rest-)Ungewissheit, die der Medizin inhärent ist, ebenso wie die Inakzeptanz von "Abwarten“ und „Beobachten“ als Strategien in der Diagnosestellung und Therapieentwicklung [21].

Manche Patientinnen empfinden die eigene potenzielle Überversorgung insgesamt eher als eine besonders gute, für- 
sorgliche und großzügige Sorge um ihre Gesundheit und fordern diese ein [21]. Trotz einer Ahnung, dass Überversorgung existiert, fürchten sie für die eigene Behandlung eher Defizite und Restriktionen [21]. Möglicherweise tendieren Angehörige und gesetzliche Stellvertreter, besonders wenn letzteren die Persönlichkeit der Patientin und der familiäre Hintergrund noch nicht ausreichend bekannt sind, eher zur Übertherapie, weil sie im Licht ihrer Pflicht zum „Wohltun“ den Vorwurf einer „Untertherapie“ befürchten $[47,49]$.

Die lebensbedrohliche Situation während des intensivmedizinischen Aufenthalts kann die Patientin und ihr Umfeld in eine emotionale Ausnahmesituation versetzen und die Entscheidungsfähigkeit beeinflussen. So kann eine Anspruchshaltung auf eine intensivmedizinische Maximaltherapie nicht nur auf fehlendem Wissen und/oder einer Fehleinschätzung der Möglichkeiten und Risiken moderner Intensivmedizin, sondern auch auf Überforderung und kognitiver Abstumpfung basieren.

Zusätzlich führen auch Kommunikationsdefizite und Schwierigkeiten bei der Interpretation übermittelter Informationen $\mathrm{zu}$ Unsicherheiten und der Forderung nach (sog.) Maximaltherapie. Zwar steht inzwischen durch den informationstechnologischen Fortschritt allen (potenziellen) Patientinnen und Angehörigen eine Fülle an Informationen zur Verfügung; oft verhindern jedoch deren fragliche Qualität und die mangelnde Gesundheitskompetenz eine individuell angemessene Einschätzung $[42,53]$. In der Informationsflut bleibt insbesondere häufig unbeachtet, dass die eingeforderte Maximaltherapie zum Überleben mit schwersten Einschränkungen führen kann [66].

\section{Ermittlung und Berücksichtigung des Patientenwillens}

Ärztliche Aufgabe ist es, die Indikation und den Patientenwillen zu einer Behandlungsentscheidung zusammenzuführen (Patientenrechtegesetz; $\S$ 1901a BGB; [17, 48]). Schon die Eruierung bzw. Interpretation des Patientenwillens auf Intensivstationen scheitert häufig an vielfältigen Problemen wie Akuität der Erkrankung, fehlende Einwilligungsfähigkeit der Patienten oder Unklarheiten über die (gesetzliche) Stellvertreterregelung und die Anwendbarkeit von Vorausverfügungen im konkreten Fall [19, 46, 47, 64]. Gespräche mit Patientinnen und Angehörigen bzw. gesetzlichen Stellvertretern sollen daher einerseits zu deren angemessener Information und Aufklärung dienen. Andererseits sollen diese Gespräche auch dazu beitragen, dass das Behandlungsteam die Legitimation diagnostischer und therapeutischer Maßnahmen durch die Patientin bzw. ihren gesetzlichen Stellvertreter regelhaft überprüft. Andernfalls kann Übertherapie resultieren. Speziell ausgebildete „communication facilitators" können bei der Bewältigung der oft komplexen Kommunikationsaufgaben behilflich sein und Übertherapie vermeiden helfen [8].

\section{Erkennen und Bewerten einer möglichen Überversorgung}

Die Verantwortung, bedarfsgerechte und ressourcenbewusste Entscheidungen aufgrund einer wissenschaftlich begründeten und individuell abgestimmten Indikationsstellung zu treffen, obliegt den behandelnden Ärztinnen [20]. Die Arzt-Patienten-Interaktion ist dabei der zentrale Ansatzpunkt, um Überversorgung zu erkennen, zu bewerten und zu vermeiden.

\section{Bewertung der Indikationsstellung und des Therapieziels unter Berücksichtigung des Patientenwillens}

Patientenwille und medizinische Indikation sind Grundlage für die Definition des übergeordneten Therapieziels [48]. Erst das Begreifen des Therapieziels als absolut maßgebend für die Indikationsstellung von Diagnostik und Therapie einerseits und die kontinuierliche Überprüfung seiner Gültigkeit unter Berücksichtigung der Gesamtheit der erhobenen Befunde und des Patientenwillens andererseits lassen Überversorgung erkennen. Die folgenden 5 Leitfragen können dabei in der Praxis (beispielsweise im Rahmen der täglichen intensivmedizinischen Visite) helfen.

Das Akronym TRIKKkann helfen, diese Fragen während der Visite oder im kollegialen Gespräch leicht zu rekapitulieren und strukturiert abzuarbeiten (• Abb. 2).

\section{Reflektiertes Entscheiden als \\ Resultat ständiger Fortbildung und eines kritischen kollegialen Austauschs}

Entscheidende Voraussetzung zur Beurteilung, ob diagnostische und therapeutische Maßnahmen geeignet sind, das Therapieziel zu erreichen, ist die Kenntnis der verfügbaren Evidenz. Neben systematisch aufgearbeiteten Informationen, Entscheidungshilfen und ggf. Leitlinien (beispielsweise im Rahmen der Initiativen „Gemeinsam klug entscheiden“ der Arbeitsgemeinschaft Wissenschaftlich Medizinischer Fachgesellschaften oder „Klug entscheiden“ der Deutschen Gesellschaft für Innere Medizin; [55, 62, 67]) spielen hierbei Zweitmeinungsverfahren eine entscheidende Rolle. Um Diagnostik und Therapie bereits so früh wie möglich und so gezielt wie möglich $\mathrm{zu}$ betreiben, können die beiden folgenden Leitfragen helfen.

\section{Allgemeine Forderungen zur Vermeidung einer Überversorgung}

Aufgrund der komplexen Natur von Überversorgung in der Intensivmedizin und ihren vielgestaltigen Einflussfaktoren und Ursachen [43] lässt sie sich nur durch eine multidimensionale Herangehensweise reduzieren und vermeiden. Das erfordert also gleichzeitig Maßnahmenbündel auf den verschiedenen Ebenen des Gesundheitssystems - also auf Mikro-, Meso- und Makroebene. Die einzelne Intensivmedizinerin mag zwar auf der normativen Ebene selbst nicht viel verändern können, ihr Einfluss auf die Leitungsebene mag eingeschränkt sein. Aber ihre professionsethische Verpflichtung, auf der Ebene des Behandlungsteams gegen Überversorgung vorzugehen und sie gegenüber den Verantwortlichen auf der Leitungsebe- 
Ein simpler TRIKK,

um Überversorgung zu erkennen und zu vermeiden...

Formuliere das

herapieziel!

R

Stelle sicher, dass für jede geplante oder laufende Therapie eine

ndikation besteht und diese geeignet ist, um das Therapieziel zu erreichen!

Stelle sicher, dass jede geplante diagnostische Prozedur eine

onsequenz hat, die den Patienten/die Patientin und das Behandlungsteam

dem Therapieziel näherbringt!

Stelle sicher, dass weiterhin mutmaßlicher, vorausverfügter oder definitiver onsens des Patienten/der Patientin für alle laufenden und geplanten

diagnostischen und therapeutischen Prozeduren besteht! e-evaluiere das Therapieziel regelmäßig und kritisch!

Abb. $2<$ Das Akronym TRIKK kann Hilfestellung sein, die bestehende Therapie und Therapieplanung regelmäBig kritisch zu überprüfenund Überversorgung zu erkennen, zu benennen und zu vermeiden

ne zu beanstanden, bleibt ausdrücklich bestehen [36].

\section{Forderungen und Empfeh- lungen auf der Ebene der Behandlungsteams (Mikroebene)}

\section{Therapieziele und -maßnahmen regelmäßig (re)evaluieren}

Ärztinnen sind in der Verantwortung, wissenschaftlich begründete und individuell abgestimmte Indikationsstellungen $\mathrm{zu}$ treffen, die sowohl angemessen als auch ressourcenbewusst sind [54]. Da$\mathrm{zu}$ bedarf es der gemeinsamen Bewertung eines medizinisch sinnvollen Behandlungsumfangs im Team und dann der Entscheidungsfindung von Patientin und Ärztin und einer kontinuierlichen und vertrauensvollen Betreuung von $\mathrm{Pa}$ tientin und Angehörigen [30, 41]. Die Frage nach der Indikation und dem Therapieziel in Übereinstimmung mit dem Patientenwillen muss nicht nur initial, sondern auch im weiteren Verlauf regelmäßig, z. B. im Rahmen der Visite oder der strukturierten Patienten- und Angehörigenkommunikation, reflektiert werden, um Überversorgung in der laufenden Therapie zu erkennen. Die getroffenen Therapieentscheidungen müssen dann für alle Beteiligten transparent und nachvollziehbar kommuniziert und dokumentiert werden [50].

\section{Vorhandene Unterstützungsange- bote für das Behandlungsteam, Patienten und Angehörige nutzen}

Verschiedene Formen klinischer Ethikberatung und Integration weiterer Spe- zialisten aus den Bereichen Palliativmedizin, klinische Psychologie/Psychosomatik, Seelsorge, Sozialdienst sind zunehmend verbreitet und sollen die Betroffenen dabei unterstützen, eine sorgfältig abgewogene und gut begründbare Entscheidung zu treffen und dabei entstehende Konflikte zu lösen [2, 9-11]. Insbesondere das Modell der proaktiven Visitenbegleitung durch klinische Ethiker oder Palliativmediziner - unter Wahrung von Schweigepflicht und Datenschutz - kann hilfreich sein, um potenzielle Überversorgung zu erkennen und gemeinsam ein neues Therapiekonzept zu erarbeiten [69-71].

\section{Patientenautonomie in der Arzt- Patienten-Beziehung fördern}

Intensivmedizinerinnen können durch die angemessene Ausgestaltung der Kommunikation mit den Patienten bzw. ihren gesetzlichen Stellvertretern und den Angehörigen deren Selbstbestimmung und Entscheidungsfähigkeit fördern, insbesondere auch im Rahmen der strukturierten Kommunikation. Dadurch können sie vielfältigen Ängsten und Unsicherheiten als bedeutsamen Einflussfaktoren für das Entstehen von Übertherapie entgegenwirken [21]. Für juristische Stellvertreter (Bevollmächtigte und Betreuer) mag es mitunter schwierig sein, eigene Interessen im Hinblick auf den Patientenwillen zu erkennen und zurückzustellen [49]. Gerade Familienangehörige befinden sich bei der Entscheidungsfindung nicht selten in einer akuten Belastungssituation, bei der sie Unterstützung durch das Be- handlungsteam erhalten sollten - sonst resultiert allzu oft eine unreflektierte Fortführung der nicht mehr indizierten lebenserhaltenden intensivmedizinischen Maßnahmen.

\section{Forderungen und Empfehlungen auf Leitungsebene (Mesoebene)}

\section{Patientenzentrierte Unterneh- menskultur im Krankenhaus leben}

Managemententscheidungen im Krankenhaus müssen sich vorrangig an qualitativ hochwertiger Patientenversorgung im Sinne des Versorgungsauftrags („ressourcenbewusste Daseinsfürsorge“; [54]) orientieren statt an betriebswirtschaftlichen Zielgrößen (z. B. Erlössteigerung). Dazu ist eine gemeinsame und unabhängige Krankenhausführung - ärztliche Direktion, Pflegedirektion, kaufmännische Leitung - erforderlich. Gemeinsam sollten Strategien entwickelt werden, die innerhalb der Organisation dazu beitragen können, Überversorgung aufzudecken (z. B. durch Implementierung von "Checklisten“) und zu vermeiden. Insbesondere müssen die Personalausstattung und die Arbeitsbedingungen den Behandlungsteams eine evidenzbasierte, patientenzentrierte Versorgung ermöglichen. Kennziffern der Unternehmenskultur werden bereits durch regelmäßige Befragungen von Mitarbeiterinnen und Patientinnen/Angehörigen erhoben. Diese Instrumente sollten zukünftig auch durch Fragen zur Wahrnehmung einer Übertherapie ergänzt werden [22].

\section{Strukturen zur Stärkung der interdisziplinären und interpro- fessionellen Kommunikation und Kooperation schaffen}

Aus organisationsethischer Perspektive ist der Krankenhausträger in der Verantwortung, strukturelle Voraussetzungen zu schaffen, die die interdisziplinäre und interprofessionelle Kommunikation und Kooperation stärken [40, 76]. Dies umfasst unter anderem die Bereithaltung von Unterstützungsstrukturen wie klinische Ethikkomitees und Palliativteams [1,2]. Eine Ressourcenumverteilung zugunsten einer angemessenen personellen Ausstat- 
Hier steht eine Anzeige.

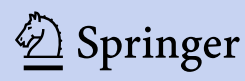


tung sowie einer nachhaltigen Qualifikation und Wertschätzung des Personals kann dem bestehenden problematischen Personalmangel gegensteuern und Übertherapie begrenzen helfen [52]. Ebenso können durch die Einführung von strukturierten - beispielsweise checklistenbasierten - Prozessen für Aufnahmen, Übergaben und Verlegungen sowie für interdisziplinäre und transsektorale $\mathrm{Ab}$ sprachen das Risiko von Komplikationen und die Gefahr von inadäquaten $\mathrm{Maß-}$ nahmen minimiert werden [24].

\section{Interdisziplinäre und interprofes- sionelle Zusammenarbeit in Aus-, Fort- und Weiterbildung stärken}

Eine adäquate berufs- und fachgruppenübergreifende Zusammenarbeit gilt als Voraussetzung für eine qualitativ hochwertige Patientenversorgung. Die Forderung zahlreicher Expertengremien (unter anderem des Wissenschaftsrats [79] und der Gesellschaft für Medizinische Ausbildung [77]) besteht deshalb in einer frühzeitigen und stetig wiederkehrenden interprofessionellen Aus-, Fort- und Weiterbildung, die sich als probates Mittel für die Verbesserung der Ergebnisqualität erwiesen hat [60]. Multiprofessionelle Fort- und Weiterbildungen kommen in der Intensivmedizin im Rahmen der Einübung von Notfallszenarien bereits vielfach zum Einsatz [57, 68]. Jenseits dieses gemeinsamen Simulationstrainings sind inter- oder multiprofessionelle Formate jedoch nach wie vor kein fester und obligatorischer Bestandteil der Fachweiterbildung bzw. Facharztausbildung in der Intensivmedizin. Die curriculare Verankerung von Themen der Über-, Unterund Fehlversorgung ist daher weiter voranzutreiben und die Umsetzung konsequent einzufordern.

\section{Forderungen und Empfeh- lungen auf gesellschaftlicher, gesundheitspolitischer und gesetzgebender Ebene (Makroebene)}

\section{Fehlanreize im Krankenhausfinan- zierungssystem minimieren}

Der Wettbewerb der Krankenhäuser trägt Merkmale ruinöser Konkurrenz, führt zu Defiziten in der Behandlungs- qualität und zu Effizienzverlusten [35]. Das Ziel des Wettbewerbs war ursprünglich die Unterbindung von Ressourcenverschwendung und die Schließung von Klinikbetten. Die Folge des Wettbewerbs ist aber eine betriebswirtschaftlich begründete Ausweitung von Leistungen unter Inkaufnahme einer illegitimen und ökonomisch sinnwidrigen Überversorgung. Grundlegende Veränderungen der Krankenhausstrukturen und der Finanzierung sind bislang ausgeblieben $[13,35]$, was als fehlender politischer Handlungswillen bewertet werden muss. Einer Debatte um eine zentrale Krankenhausbedarfsplanung und eine Priorisierung in der Gesundheitsversorgung, die in anderen Ländern Europas offen geführt wird, hat sich die deutsche Gesundheitspolitik bislang konsequent entzogen.

Die Anwendung des fallpauschalbasierten Vergütungssystems bedarf - nicht nur aus Sicht der Intensivmedizin und -pflege - dringend einer Reformierung. Anreize zur Fallzahlsteigerung und Leistungsausweitung aus ökonomischen Gründen sollten nicht weiterhin gesetzt werden. Konservative und interventionelle/operative Medizin sollten gleich bewertet werden [54, 63]. Ziel muss es sein, den ökonomischen Druck aus der Beatmungstherapie und anderen invasiven intensivmedizinischen Prozeduren zu nehmen, um bei der Entscheidung für oder gegen eine bestimmte Therapiemaßnahme die Abwägung des Patientenwillens mit der Indikation in den Mittelpunkt stellen zu können [63]. Fehlanreize für Übertherapie könnten so gezielt minimiert werden. Die Bedeutung und Wertschätzung einer kritischen ethischen Reflexion im Behandlungsteam sollte sich auch darin ausdrücken, dass sich definierte Formen von Ethikberatung im Vergütungssystem abbilden.

\section{Gesellschaftlichen Diskurs zur Überversorgung anstoßen}

Die Vermeidung einer Überversorgung erfordert auch ein Umdenken innerhalb der Gesellschaft, die weithin von einer ausgeprägten Vorstellung der Omnipotenz der Intensivmedizin gekennzeichnet ist und daraus umfassende
(Versorgungs-)Ansprüche ableitet. Es gilt deshalb, auch in der Allgemeinbevölkerung einen umfassenden Diskurs über die Möglichkeiten und Grenzen von Intensivmedizin anzuregen und qualifiziert zu begleiten. Dazu ist auch ein Diskurs über unrealistische Gesundheitsideale und unreflektierte Optimierungsvorstellungen erforderlich. Anreize sollten eher der Gesunderhaltung und dem gelingenden Umgang mit Alter(n), Krankheit und Beeinträchtigungen dienen als einer nachträglichen Reparatur von Organen und Körperfunktionen.

\section{Umfassende Vorsorgeplanung einführen, ausweiten und evaluieren}

Vorausverfügte Willensäußerungen (schriftlich oder mündlich) und die Benennung von Vorsorgebevollmächtigten können jedem einzelnen (potenziellen) Patienten helfen, ungewünschte intensivmedizinische Maßnahmen zu verhindern. Schwierigkeiten mit konventionellen Patientenverfügungen und deren Anwendung auf der Intensivstation sind durch zahlreiche empirische Untersuchungen aufgedeckt worden [32]. Das Konzept des Advance Care Planning (ACP), das die vorhandenen Schwachstellen adressiert [23, 83], ist jedoch bislang nicht flächendeckend und auf allen relevanten Ebenen realisiert. Auch die Voraussetzungen für die Kostenübernahme für ACP sind gesetzlich nur für ausgewählte Bereiche geklärt (vgl. \$132g SGB V). Vor allem für Intensivmedizinerinnen könnte aus einer breiteren, sektorenübergreifenden Einführung mehr Handlungssicherheit im Einzelfall resultieren, die dazu beitragen kann, Übertherapie zu vermeiden und eine Therapie durchzuführen, die an den tatsächlichen individuellen Bedürfnissen der Patientin orientiert ist.

\section{Zusammenfassung}

Überversorgung, also eine unangemessen umfangreiche Therapie, ist ein ernstzunehmendes negatives Phänomen auch auf Intensivstationen im deutschen $\mathrm{Ge}$ sundheitssystem. Sie kann einerseits Patienten, Angehörigen und den Behandlungsteams schaden und führt anderer- 
seits durch Ressourcenverschwendung zum Schaden für die Allgemeinheit.

Überversorgung ist einem Konglomerat mehrerer Ursachen geschuldet. Wesentlicher Faktor ist die Verquickung von unbedachter, unangemessener oder fehlerhafter Indikationsstellung mit falschen Vorstellungen und Erwartungen in Bezug auf Nutzen und Risiken intensivmedizinischer Behandlungsmaßnahmen, insbesondere auf Seiten der Patientinnen oder ihrer gesetzlichen Stellvertreter.

Auf normativer Ebene sind gesellschafts- und gesundheitspolitische Fehlentwicklungen $\mathrm{zu}$ korrigieren, die allerdings auf der Ebene der Behandlungsteams kaum zu beeinflussen sind. Umso mehr bleibt es berufsethische ärztliche Verpflichtung, ausgehend vom medizinisch sinnvoll Möglichen im Behandlungsteam einen angemessenen Behandlungsumfang für jede Patientin individuell festzulegen, der deren Willen bestmöglich entspricht. Im vorliegenden Positionspapier sind im Akronym TRIKK 5 Leitfragen für intensivmedizinische Behandlungsteams zusammengefasst, die dabei helfen können, Überversorgung $\mathrm{zu}$ erkennen und $\mathrm{zu}$ reduzieren - bestenfalls sogar zu vermeiden.

Die dargestellten Argumente und Forderungen haben auch Bestand während außergewöhnlicher Belastungen wie der aktuellen Pandemie oder zukünftiger Katastrophenfälle. Notfallsituationen setzen Grundregeln für medizinische Entscheidungen nicht außer Kraft. Die COVID-19-Pandemie hat nachdrücklich gezeigt, dass es geboten sein kann, für besondere Situationen eine Notfallreserve bereitzuhalten und die dafür notwendige Finanzierung durchgängig $\mathrm{zu}$ sichern. Dabei müssen allerdings räumliche, apparative, logistische und insbesondere personelle Ressourcen bedacht werden. Zugleich ist darauf zu achten, dass eine solcherart vorgehaltene Reserve nicht eine Angebotsausweitung begünstigt, die wiederum einer Überversorgung Vorschub leisten kann.

\section{Korrespondenzadresse}

Prof. Dr. med. Uwe Janssens

Klinik für Innere Medizin und Internistische Intensivmedizin, St.-Antonius-Hospital Eschweiler

Dechant-Deckers-Str. 8, 52249 Eschweiler,

Deutschland

uwe.janssens@sah-eschweiler.de

\section{Einhaltung ethischer Richtlinien}

Interessenkonflikt. A. Michalsen, G. Neitzke, J. Dutzmann, A. Rogge, A.-H. Seidlein, S. Jöbges, H. Burchardi, C. Hartog, F. Nauck, F. Salomon, G. Duttge, G. Michels, K. Knochel, S. Meier, P. Gretenkort und U. Janssens geben an, dass kein Interessenkonflikt besteht.

Für diesen Beitrag wurden von den Autoren keine Studien an Menschen oder Tieren durchgeführt. Für die aufgeführten Studien gelten die jeweils dort angegebenen ethischen Richtlinien.

Open Access. Dieser Artikel wird unter der Creative Commons Namensnennung 4.0 International Lizenz veröffentlicht, welche die Nutzung, Vervielfältigung, Bearbeitung, Verbreitung und Wiedergabe in jeglichem Medium und Format erlaubt, sofern Sie den/die ursprünglichen Autor(en) und die Quelle ordnungsgemäß nennen, einen Link zur Creative Commons Lizenz beifügen und angeben, ob Änderungen vorgenommen wurden.

Die in diesem Artikel enthaltenen Bilder und sonstiges Drittmaterial unterliegen ebenfalls der genannten Creative Commons Lizenz, sofern sich aus der Abbildungslegende nichts anderes ergibt. Sofern das betreffende Material nicht unter der genannten Creative Commons Lizenz steht und die betreffende Handlung nicht nach gesetzlichen Vorschriften erlaubt ist, ist für die oben aufgeführten Weiterverwendungen des $\mathrm{Ma}$ terials die Einwilligung des jeweiligen Rechteinhabers einzuholen.

Weitere Details zur Lizenz entnehmen Sie bitte der Lizenzinformation auf http://creativecommons.org/ licenses/by/4.0/deed.de.

\section{Literatur}

1. Adler K, Schlieper D, Kindgen-Milles D et al (2017) Integration der Palliativmedizin in die Intensivmedizin : Systematische Ubersichtsarbeit Anaesthesist 66:660-666

2. Au SS, Couillard P, Roze des Ordons A et al (2018) Outcomes of ethics consultations in adult ICus: a systematic review and meta-analysis. Crit Care Med 46:799-808

3. Beinersdorf A (2007) Der Informationsverlust bei der Patientenverlegung. intensiv 15:268-273

4. Benoit DD, Jensen HI, Malmgren J et al (2018) Outcome in patients perceived as receiving excessive care across different ethical climates: a prospective study in 68 intensive care units in Europe and the USA. Intensive Care Med 44:1039-1049
5. Bion J, Antonelli M, Blanch L et al (2018) White paper: statement on conflicts of interest. Intensive Care Med 44:1657-1668

6. Boothroyd LJ, Spaziano M, Guertin JR et al (2013) Transcatheter aortic valve implantation: recommendations for practice based on a multidisciplinary review including cost-effectiveness and ethical and organizational issues. Can J Cardiol 29:718-726

7. Consortium WHOST, Pan H, Peto R et al (2020) Repurposed antiviral drugs for Covid-19-interim WHO solidarity trial results. N Engl J Med. https:// doi.org/10.1056/NEJMoa2023184

8. Curtis JR, Treece PD, Nielsen EL et al (2016) Randomized trial of communication facilitators to reduce family distress and intensity of end-of-life care. Am J Respir Crit Care Med 193:154-162

9. Deffner T, Michels G, Nojack A et al (2020) Psychologische Versorgung auf der Intensivstation : Tatigkeitsbereiche, Aufgaben, Anforderungen und Ausstattung. Med Klin Intensivmed Notfmed 115:205-212

10. DeffnerT, Rosendahl J, Niecke A (2020) Psychotraumatologische Aspekte in der Intensivmedizin. Med Klin Intensivmed Notfmed 115:511-518

11. Deffner T, Schwarzkopf D, Waydhas $C$ et al (2019) Psychologische Versorgung auf deutschen Intensivstationen:Ergebnisse einer Umfrage unter den Mitgliedern der Deutschen Interdisziplinaren Vereinigung fur Intensiv- und Notfallmedizin. Med Klin Intensivmed Notfmed. https://doi.org/10. 1007/s00063-019-00638-2

12. Deutscher Ethikrat (2014) Vom Krankenhaus zum kranken Haus? Klinikalltag zwischen ethischem Anspruch und Kostendruck. https://www.ethikrat. org/weitere-veranstaltungen/vom-krankenhauszum-kranken-haus-klinikalltag-zwischenethischem-anspruch-und-kostendruck/. Zugegriffen:23. Aug. 2020

13. Deutscher Ethikrat (2011) Nutzen und Kosten im Gesundheitswesen - Zur normativen Funktion ihrer Bewertung. https://www.ethikrat. org/fileadmin/Publikationen/Stellungnahmen/ deutsch/DER_StnAllo-Aufl2_Online.pdf. Zugegriffen:23.Aug. 2020

14. Druml W, Druml C (2019) Ubertherapie in der Intensivmedizin. Med Klin Intensivmed Notfmed 114:194-201

15. Duttge G, Er D, Fischer ES (2016) Vertrauen durch Recht? In: Steinfath H, Wiesemann C (Hrsg) Autonomie und Vertrauen - Schlüsselbegriffe der modernen Medizin. Springer, Berlin, S239-291

16. Eddy DM (1984) Variations in physician practice: the role of uncertainty. Health Aff 3:74-89

17. Elshaug AG, Rosenthal MB, Lavis JN et al (2017) Levers for addressing medical underuse and overuse: achieving high-value health care. Lancet 390:191-202

18. Fleischmann-Struzek C, Mikolajetz A, Reinhart K et al (2019) Hospitalisierung und Intensivtherapie am Lebensende. Dtsch Arztebl Int 116:653-660

19. Grignoli N, Di Bernardo V, Malacrida R (2018) New perspectives on substituted relational autonomy for shared decision-making in critical care. Crit Care 22:260

20. Grote Westrick M, Volbracht E (2020) Überversorgung - Ausmaß, Ursachen und Gegenmaßnahmen.GGW 20:7-15

21. Hambrock U (2019) Erfahrungen mit Überversorgung: Qualitativ-psychologische Studie mit Patienten und Ärzten der Bertelsmann Stiftung. https://www.bertelsmann-stiftung.de/de/ publikationen/publikation/did/erfahrungen-mitueberversorgung/.Zugegriffen:7.Dez. 2020 
22. Hartog CS, Hoffmann F, Mikolajetz A et al (2018) Ubertherapie und emotionale Erschopfung in der "end-of-life care" : Ergebnisse einer Mitarbeiterumfrage auf der Intensivstation. Anaesthesist 67:850-858

23. Hartog CS, Spies CD, Michl S et al (2020) Advance Care Planning in Zeiten der Corona-Pandemie eine Chance fur die Patientenautonomie in der Akutsituation. Med Klin Intensivmed Notfmed 115:571-572

24. Haynes AB, Weiser TG, Berry WR et al (2009) A surgical safety checklist to reduce morbidity and mortality in a global population. $\mathrm{N}$ Engl J Med 360:491-499

25. Hofmann B (2019) Expanding disease and undermining the ethos of medicine. Eur $J$ Epidemiol 34:613-619

26. Hofmann B (2018) Looking for trouble? Diagnostics expanding disease and producing patients. J Eval Clin Pract 24:978-982

27. Ilkilic I (2008) Kulturelle Aspekte bei ethischen Entscheidungen am Lebensende und interkulturelle Kompetenz. Bundesgesundheitsblatt Gesundheitsforschung Gesundheitsschutz 51:857-864

28. Klemperer D (2008) Gefahr für das ärztliche Urteilsvermögen ocesses. Dtsch Arztebl Int 105:419-420 (author reply 421-412)

29. Kohn R, Rubenfeld GD, Levy MM et al (2011) Rule of rescue or the good of the many? An analysis of physicians' and nurses' preferences for allocating ICU beds. Intensive Care Med 37:1210-1217

30. Kon AA, Davidson JE, Morrison W et al (2016) Shared decision making in ICus: an American College of Critical Care Medicine and American Thoracic Society policy statement. Crit Care Med 44:188-201

31. Kopp R, Wildenauer R, Marx G (2020) Umsichtig und vernünftig handeln auf der operativen Intensivstation. Anasth Intensivmed 61:466-471

32. Langer S, Knorr J-U, Berg A (2013) Umgang mit Patientenverfügungen: Probleme durch pauschale Formulierungen. Dtsch Arztebl Int 110:2186-2188

33. Leopoldina Nationale Akademie der Wissenschaften (2016) Zum Verhältnis von Medizin und Ökonomie im deutschen Gesundheitssystem: 8 Thesen zur Weiterentwicklung zum Wohle der Patienten und der Gesellschaft. https://www.leopoldina. org/publikationen/detailansicht/publication/ zum-verhaeltnis-von-medizin-und-oekonomieim-deutschen-gesundheitssystem-2016/.Zugegriffen:23. Aug. 2020

34. Long AC, Brumback LC, Curtis JR et al (2019) Agreement with consensus statements on end-oflife care: a description of variability at the level of the provider, hospital, and country. Crit Care Med 47:1396-1401

35. Loos S, Albrecht M, Zich K (2019) Zukunftsfähige Krankenhausversorgung: Simulation und Analyse einer Neustrukturierung der Krankenhausversorgung am Beispiel einer Versorgungsregion in Nordrhein-Westfalen. https://www.bertelsmannstiftung.de/de/publikationen/publikation/did/ zukunftsfaehige-krankenhausversorgung. Zugegriffen:23. Aug. 2020

36. Maio G (2009) Dienst am Menschen oder KundenDienst? Ethische Grundreflexionen zur sich wandelnden ärztlichen Identität. In: Katzenmeier C, Bergdolt K (Hrsg) Das Bild des Arztes im 21. Jahrhundert. Springer, Berlin, S21-35

37. Marshall AP, Tobiano G, Murphy N et al (2019) Handover from operating theatre to the intensive care unit: A quality improvement study. Aust Crit Care 32:229-236
38. McGauran N, Wieseler B, Kreis J et al (2010) Reporting bias in medical research - a narrative review. Trials 11:37

39. Metaxa V, Ely EW (2020) Cultural diversity. In: Michalsen A, Sadovnikoff N (Hrsg) Compelling ethical challenges in critical care and emergency medicine. Springer, Berlin, S49-55

40. Michalsen A, Hillert A (2018) Stressreduktion und Burn-out-Prophylaxe. In: Kluge S, Marx C, Janssens $\mathrm{U}$, Zacharowski K (Hrsg) Management in der Intensivmedizin. Medizinisch Wissenschaftliche Verlagsgesellschaft, Berlin, S183-188

41. Michalsen A, Long AC, DeKeyser Ganz F et al (2019) Interprofessional shared decision-making in the ICU: a systematic review and recommendations from an expert panel. Crit Care Med 47:1258-1266

42. Moorhead SA, Hazlett DE, Harrison L et al (2013) A new dimension of health care: systematic review of the uses, benefits, and limitations of social media for health communication. J Med Internet Res 15:e85

43. Morgan DJ, Leppin AL, Smith CD et al (2017) A practical framework for understanding and reducing medical overuse: conceptualizing overuse through the patient-clinician interaction. J Hosp Med 12:346-351

44. Moss M, Good VS, Gozal D et al (2016) An official critical care societies collaborative statement: burnout syndrome in critical care health care professionals: a call for action. Am J Crit Care 25:368-376

45. Müller R, Ganten D, Larisch J (2014) Public Health: Gesundheit ist mehr als Medizin. Dtsch Arztebl Int 111:1900

46. Mulley AG, TrimbleC, Elwyn G (2012) Stop the silent misdiagnosis: patients' preferences matter. BMJ 345:e6572

47. Neitzke G (2019) Ermittlung des Patientenwillens. Anasthesiol Intensivmed Notfallmed Schmerzther 54:474-483

48. Neitzke G (2014) Indikation: fachliche und ethische Basis ärztlichen Handelns. Med Klin Intensivmed Notfmed 109:8-12

49. Neitzke G (2019) Juristische Stellvertreter in der Medizin: Bevollmächtigte und Betreuer. Anasthesiol Intensivmed Notfallmed Schmerzther 54:485-494

50. Neitzke G, Boll B, Burchardi H et al (2017) Dokumentation Therapiebegrenzung-Empfehlung der Sektion Ethik der Deutschen Interdisziplinäre Vereinigung für Intensiv und Notfallmedizin (DIVI) unter Mitarbeit der Sektion Ethik der Deutschen Gesellschaft für Internistische Intensivmedizin und Notfallmedizin (DGIIN). Med Klin Intensivmed Notfmed 112:527-530

51. Neitzke G, Burchardi H, Duttge G et al (2016) Grenzen der Sinnhaftigkeit von Intensivmedizin. Med Klin Intensivmed Notfmed 111:486-492

52. Neuraz A, Guerin C, Payet C et al (2015) Patient mortality is associated with staff resources and workload in the ICU: a multicenter observational study. Crit Care Med 43:1587-1594

53. Noordman J, van Vliet L, Kaunang M et al (2019) Towards appropriate information provision for and decision-making with patients with limited health literacy in hospital-based palliative care in Western countries: a scoping review into available communication strategies and tools for healthcare providers. BMC Palliat Care 18:37

54. Nothacker M, Busse R, Elsner P et al (2019) Medizin und Okonomie: Massnahmen fur eine wissenschaftlich begrundete, patientenzentrierte und ressourcenbewusste Versorgung. Ein Strategiepapier der Arbeitsgemeinschaft der Wissenschaftli- chen Medizinischen Fachgesellschaften (AWMF). Dtsch Med Wochenschr 144:990-996

55. Nothacker M, Kreienberg R, Kopp IB (2017) "Gemeinsam Klug Entscheiden" - eine Initiative der AWMF und ihrer Fachgesellschaften: Mission, Methodik und Anwendung. Z Evid Fortbild Qual Gesundhwes 129:3-11

56. OECD (2017) Tackling wasteful spending on health. OECD Publishing, Paris

57. Partecke M, Balzer C, Finkenzeller I et al (2016) Interprofessionelles Lernen an der Universitätsmedizin Greifswald - Didaktische Konzeption und praktische Etablierung eines notfallmedizinischen Teamtrainings von Medizinstudierenden und Aus zubildenden derGesundheits-und Krankenpflege. GMS J Med Educ 33:Doc32

58. Pfister H-R, Jungermann H, Fischer K (2016) Die Psychologie der Entscheidung. Spektrum Akademischer Verlag, Berlin

59. Ranieri VM, Thompson BT, Barie PS et al (2012) Drotrecogin alfa (activated) in adults with septic shock. N Engl J Med 366(22):2055-2064. https:// doi.org/10.1056/NEJMoa120229

60. Reeves S, Perrier L, Goldman J et al (2013) Interprofessional education: effects on professional practice and healthcare outcomes. Cochrane Database Syst Rev. https://doi.org/10.1002/14651858. CD002213.pub3:CD002213

61. Riessen R, Hermes C, Bodmann KF et al (2018) Vergutung intensivmedizinischer Leistungen im DRG-System : Aktuelle Probleme und Losungsvorschlage. Med Klin Intensivmed Notfmed 113:13-23

62. Riessen R, Kluge S, Janssens U et al (2017) Klug entscheiden - Empfehlungen in der internistischen Intensivmedizin. Internist 58:550-555

63. Riessen R, Markewitz A, Grigoleit $M$ et al (2020) Diskussionspapier fur eine Reform der Krankenhausfinanzierung in Deutschland aus der Perspektive der Intensivmedizin. Med Klin Intensivmed Notfmed 115:59-66

64. Robertsen A, Jöbges S, Sadovnikoff N (2020) Consent, advance directives, and decision by proxies. In: Michalsen A, Sadovnikoff N (Hrsg) Compelling ethical challenges in critical care and emergency medicine. Springer, Berlin, S35-47

65. Sachverständigenrat zur Begutachtung der Entwicklung im Gesundheitswesen (SVR) (2018) Bedarfsgerechte Steuerung der Gesundheitsversorgung. https://www.svr-gesundheit.de/index. php?id=606.Zugegriffen: 7 . Dez. 2020

66. Sadoughi F, Nasiri S, Ahmadi H (2018) The impact of health information exchange on healthcare quality and cost-effectiveness: a systematic literature review. Comput Methods Programs Biomed 161:209-232

67. Schaefer C, Klemperer D (2020) Mit Leitlinien, Shared Decision Making und Choosing Wisely gegen Über-, Unter- und Fehlversorgung? GGW 20:23-30

68. Schindele D, Muller-Wolff T, McDonough JP et al (2020) Klinische Handlungskompetenzen gemeinsam verbessern -interprofessionelles Lernen in der Intensivmedizin. Med Klin Intensivmed Notfmed 115:545-549

69. Schneiderman LJ (2006) Effect of ethics consultations in the intensive care unit. Crit Care Med 34:S359-S363

70. Schneiderman LJ (2005) Ethics consultation in the intensive care unit. Curr Opin Crit Care 11:600-604

71. Schneiderman LJ, Gilmer T, Teetzel HD (2000) Impact of ethics consultations in the intensive care setting: a randomized, controlled trial. Crit Care Med 28:3920-3924 
Hier steht eine Anzeige.

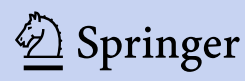


72. Schreyögg J, Milstein R (2020) Bedarfsgerechte Gestaltung der Krankenhausvergütung - Reformvorschläge unter der Berücksichtigung von Ansätzen anderer Staaten. https://www.hche.uni-hamburg. de/search.html?q=Bedarfsgerechte+Gestaltung. Zugegriffen: 7.Dez. 2020

73. Schweda M, Schicktanz S, Raz A et al (2017) Beyond cultural stereotyping:views on end-of-life decision making among religious and secular persons in the USA, Germany, and Israel. BMC Med Ethics 18:13

74. Sprung $C L$, Jennerich $A L$, Joynt $G M$ et al (2020) The influence of geography, religion, religiosity and institutional factors on worldwide end-of-life care for the critically ill: the WELPICUS study. J Palliat Care

75. Stüwe H (2015) Private Klinikträger: Die Großen erzielen gute Gewinne. Dtsch Arztebl Int 112:1056

76. Van den Bulcke B, Metaxa V, Reyners AKet al (2020) Ethical climate and intention to leave among critical care clinicians: an observational study in 68 intensive care units across Europe and the United States. Intensive Care Med 46:46-56

77. Walkenhorst U, Mahler C, Aistleithner Ret al (2015) Positionspapier GMA-Ausschuss - Interprofessionelle Ausbildung in den Gesundheitsberufen. GMS ZMed Ausbild 32:Doc22

78. Wieland W (1986) Strukturwandel der Medizin und ärztliche Ethik. Philosophische Überlegungen zu Grundfragen einer praktischen Wissenschaft. Carl Winter, Heidelberg

79. Wissenschaftsrat (2014) Empfehlungen zur Weiterentwicklung des Medizinstudiums in Deutschland auf Grundlage einer Bestandsaufnahme der humanmedizinischen Modellstudiengänge. https://www.wissenschaftsrat.de/download/ archiv/4017-14.pdf.Zugegriffen: 7. Dez. 2020

80. Wohlmannstetter M (2019) Überpflege - gibt es das auch? Med Klin Intensivmed Notfmed 114:202-206

81. Wray CM, Loo LK (2015) The diagnosis, prognosis, and treatment of medical uncertainty. J Grad Med Educ 7:523-527

82. Zander B, Dobler L, Baumler Metal (2014) Implizite Rationierung von Pflegeleistungen in deutschen Akutkrankenhausern - Ergebnisse der internationalen Pflegestudie RN4Cast. Gesundheitswesen 76:727-734

83. Zentrale Ethikkommission (2020) Stellungnahme der Zentralen Kommission zur Wahrung ethischer Grundsätze in der Medizin und ihren Grenzgebieten (Zentrale Ethikkommission) bei der Bundesärztekammer: „Außerklinische Ethikberatung“. Dtsch Arztebl Int 117:A628

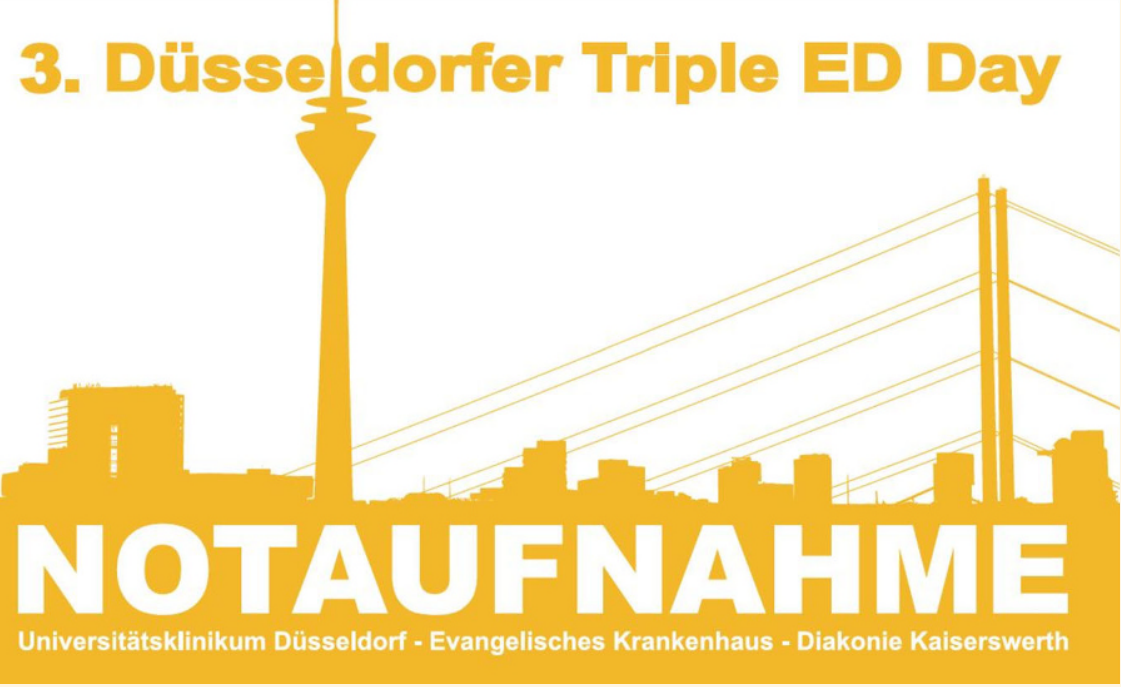

\section{Düsseldorfer „Triple ED Day“ am 11.09.2021}

Regelmäßige Fortbildungen sind wichtig, gerade wenn es um Notfallmedizin geht. Der Düsseldorfer "Triple ED Day" gibt uns die Möglichkeit, eine klinikübergreifende Fortbildung rund um Notfallmedizin und Zentrale Notaufnahmen virtuell zu begehen. Ziel ist es, aktuelles notfallmedizinisches Wissen mit besonderem Fokus auf die Zentrale Notaufnahme praxisorientiert zu vermitteln.

Neben einem Update zur Versorgung einzelner Krankheitsbilder wollen wir dabei insbesondere der aktuellen Entwicklung interdisziplinärer Zentraler Notaufnahmen Rechnung tragen. Da in der Notfallmedizin PatientInnen nicht mit einer Diagnose, sondern mit einem Symptom vorstellig werden, verfolgen wir in unserer Fortbildung einen leitsymptom- und fallorientierten Ansatz.

Da Notfallmedizin nur im Team funktionieren kann, laden wir neben den in den Zentralen Notaufnahmen tätigen pflegerischen und ärztlichen Mitarbeitenden auch Kolleginnen und Kollegen aller klinischen Partner und die Mitarbeitenden des Rettungs- und Notarztdienstes ein. Notfallmedizinisch interessierte Studierende sind ebenfalls herzlich willkommen. Durch die Chatfunktion des OnlineFormates ergibt sich Gelegenheit für Fragen und spannende Diskussionen der Vorträge. Wir würden uns sehr freuen, Sie bei unserer interprofessionellen und interdisziplinären Veranstaltung online begrüßen zu dürfen!

\section{Wissenschaftliche Leitung:}

Prof. Dr. Michael Bernhard, Düsseldorf

Alexander Kleophas, Düsseldorf

Martin Pin, Düsseldorf

\section{Organisation:}

Universitätsklinikum Düsseldorf

Zentrale Notaufnahme

Moorenstr. 5

40225 Düsseldorf

\section{Teilnahme:}

Der Düsseldorfer Triple ED Day wird über die bekannte Fortbildungsplattform NOW TO GO übertragen.

Teilnehmen kann jeder, der sich kostenlos unter www.nowtogo.de registriert hat.

Die Veranstaltung ist kostenfrei. Die Anerkennung der Fortbildungsveranstaltung wurde bei der Ärztekammer Berlin und bei der Registrierung berufl ich Pflegender beantragt. 